\title{
TRPM7 inhibitor carvacrol protects brain from neonatal hypoxic-ischemic injury
}

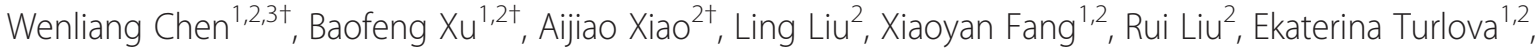 \\ Andrew Barszczyk ${ }^{2}$, Xiao Zhong ${ }^{1,2}$, Christopher L F Sun ${ }^{5}$, Luiz R G Britto ${ }^{6}$, Zhong-Ping Feng ${ }^{2^{*}}$ \\ and Hong-Shuo Sun ${ }^{1,2,3,4^{*}}$
}

\begin{abstract}
Background: Our previous study found that suppression of TRPM7 reduced neuronal death in adult rat ischemic brain injury. It was reported that carvacrol blocked TRPM7 and attenuated brain injury in an adult rat MCAO model. The effects of carvacrol on neonatal stroke remain unknown. This study investigated the effects of carvacrol on neuronal injury and behavioral impairment after hypoxia-ischemia in neonatal mice and the potential signaling pathway underlying these effects.

Results: Carvacrol inhibited TRPM7 current in HEK293 cells over-expressing TRPM7 and TRPM7-like current in hippocampal neurons in a dose-dependent manner. Carvacrol (>200 $\mu \mathrm{M})$ reduced OGD-induced neuronal injury in cortical neurons. 24 hours after HI, TRPM7 protein level in the ipsilateral hemisphere was significantly higher than in the contralateral hemisphere. Carvacrol (30 and $50 \mathrm{mg} / \mathrm{kg}$ ) pre-treatment reduced brain infarct volume 24 hours after $\mathrm{HI}$ in a dose-dependent manner. Carvacrol pre-treatment also improved neurobehavioral outcomes. Furthermore, animals pre-treated with carvacrol had fewer TUNEL-positive cells in the brain compared to vehicle-treated animals 3 days after $\mathrm{HI}$. Carvacrol pre-treatment also increased Bcl-2/Bax and p-Akt/t-Akt protein ratios and decreased cleaved caspase-3 protein expression 24 hours after $\mathrm{HI}$.
\end{abstract}

Conclusions: Carvacrol pre-treatment protects against neonatal hypoxic-ischemic brain injury by reducing brain infarct volume, promoting pro-survival signaling and inhibiting pro-apoptotic signaling, as well as improving behavioral outcomes. The neuroprotective effect may be mediated by the inhibition of TRPM7 channel function. Carvacrol is a potential drug development target for the treatment of neonatal stroke.

Keywords: TRPM7, Neonatal hypoxic-ischemic brain injury, Neuroprotection, Carvacrol

\section{Background}

Hypoxic-ischemic injury or stroke in mammalian brains elicits delayed neuronal death (DND) [1]. Previous studies have considered glutamate excitotoxicity as a key mechanism in stroke [2]. However, the inhibition of glutamate signaling has not been sufficiently neuroprotective in clinical trials [3]. Recently, non-glutamate mechanisms have attracted more attention in stroke research. Nonglutamate mechanisms also lead to intracellular ionic

\footnotetext{
*Correspondence: zp.feng@utoronto.ca; hss.sun@utoronto.ca

${ }^{\dagger}$ Equal contributors

${ }^{2}$ Department of Physiology, Faculty of Medicine, University of Toronto, 1

King's College Circle, Toronto M5S 1A8, Canada

'Department of Surgery, Faculty of Medicine, University of Toronto, 1 King's

College Circle, Toronto M5S 1A8, Canada

Full list of author information is available at the end of the article
}

imbalance and neuronal cell death in ischemia and stroke [4]. TRPM7 is an important non-glutamate mechanism and thus a potential target for drug development against ischemic brain injury [4].

Transient receptor potential melastatin 7 (TRPM7) belongs to the melastatin-related subfamily of TRP channels and is ubiquitously expressed in almost all tissues, including brain [5]. TRPM7 is a $\mathrm{Ca}^{2+}$-permeable, nonselective cation channel that has recently gained attention as a potential cation influx pathway involved in ischemic neuronal injury. An earlier study has demonstrated that TRPM7 is a key mediator of anoxic neuronal death [6,7]. Subsequent reports have revealed that TRPM7 mRNA and protein expression increase in the brain after cerebral ischemic injury and in hippocampal neurons subjected to 
oxygen-glucose deprivation (OGD) [7-10]. Moreover, it is found that Lactuside B and Ginsenoside-Rd protect against cerebral ischemic damage, at least in part by decreasing TRPM7 expression. In vivo study has shown that TRPM7 suppression by virally mediated gene silencing prevents delayed neuronal cell death and promotes neurobehavioral functional recovery in a rat global cerebral ischemia model [11]. Hence, TRPM7 seems to be a promising potential therapeutic target for drug development for stroke.

Carvacrol, a pungent natural compound often used as a food additive, has been reported to block TRPM7 current in HEK cells over-expressing TRPM7 and in hippocampal neurons [12], as well as provide neuroprotection in adult mice subjected to focal ischemia [13]. Carvacrol is therefore a potential pharmacological tool for studying the functions of TRPM7 channels in vitro and in vivo.

Neonatal hypoxic-ischemic (HI) brain injury (also known as neonatal stroke) and its related brain disorders hypoxic-ischemic encephalopathy (HIE) and cerebral palsy, are a major cause of morbidity and mortality in infants and children, with a reported incidence of 2-9 per 1000 births [14]. Approximately $20-50 \%$ of infants that have suffered HI will die and up to $25 \%$ will suffer permanent brain damage [14]. It causes severe neurological disorders and lifelong disability in survivors, including cerebral palsy, mental retardation, and epilepsy [15]. Currently, there are no effective pharmacological interventions available for protecting neonatal brains after $\mathrm{HI}$ injury. The effect of carvacrol in neonatal stroke has not yet been studied. Here we report the neuroprotective effects of carvacrol in mouse neonatal hypoxic-ischemic brain injury.

\section{Results}

Carvacrol inhibits TRPM7 currents in HEK293 cells and hippocampal neurons

First, we performed whole-cell patch-clamp experiments to examine the effect of carvacrol on TRPM7 current in HEK293 cells over-expressing TRPM7 and on TRPM7-like native currents in mouse hippocampal neurons. Carvacrol inhibited TRPM7 current in HEK293 cells over-expressing TRPM7 (Figure 1A, $\mathrm{n}=6$ cells) and TRPM7-like currents in mouse primary hippocampal neurons (Figure $1 \mathrm{~B}, \mathrm{n}=6$ cells) in a dose-dependent manner. Thus, carvacrol is a valid pharmacological tool for further in vitro and in vivo studies.

\section{Carvacrol protects neurons from OGD-induced cell injury in vitro}

We next tested whether carvacrol can protect neurons from anoxic insult (oxygen and glucose deprivation: OGD) in vitro. Propidium iodide (PI) fluorescence intensity in cortical neurons was significantly greater following OGD. We showed that carvacrol pre-incubation for 30 minutes significantly reduced PI fluorescence intensity in a dosedependent manner (200-800 $\mu \mathrm{M}$ concentration) compared to that of vehicle-treat group. The PI fluorescence intensity was reduced by $28.74 \%$ at $200 \mu \mathrm{M}, 54.43 \%$ at $500 \mu \mathrm{M}$ and $48.59 \%$ at $800 \mu \mathrm{M}$ carvacrol (Figure $1 \mathrm{C}, \mathrm{p}<0.05$ for all three doses, $\mathrm{n}=5$ ). This result was further confirmed by double-staining with PI and neuronal nuclei (NeuN) antibody to evaluate the percentage of PI positive cells. As shown in Figure 1D and E, the percentage of PI positive cells was $12.7 \pm 1.5 \%$ in the control group, and this increased significantly to $40.1 \pm 3.1 \%$ in the OGD group (", $\mathrm{p}<0.05, \mathrm{n}=4$ ), but was significantly less with $24.7 \pm$ $2.8 \%$ in the carvacrol pre-treatment group $(300 \mu \mathrm{M}$, \#, $\mathrm{p}<0.05, \mathrm{n}=4)$. This in vitro data indicates that carvacrol is able to protect cultured neurons from anoxic insult.

\section{Carvacrol pre-treatment attenuates infarct volume of hypoxic-ischemic injury in vivo in a dose-dependent manner}

Next, we asked whether carvacrol can reduce brain damage in vivo using a mouse neonatal hypoxic-ischemic brain injury model. We observed that carvacrol pretreatment (30 and $50 \mathrm{mg} / \mathrm{kg}$ i.p., $30 \mathrm{~min}$ before $\mathrm{HI}$ ) significantly reduced infarct volume 24 hours after HI. TTC staining of coronal sections of mouse brains was used for evaluating the infarct volume. Representative images of TTC staining were shown in Figure 2A, where white areas indicated brain damage. There was no detectable infarction in the sham group (data not shown). Infarct volume in the vehicle-treated HI group (Vehicle) was $57.83 \pm 5.18 \%$ ( $n=24$ pups). Carvacrol pre-treatment (30 and $50 \mathrm{mg} / \mathrm{kg}$ ) significantly reduced the infarct volume to $31.11 \pm 7.63 \% \quad(\mathrm{n}=11$ pups $)$ and $6.18 \pm 3.73 \% \quad(\mathrm{n}=17$ pups), respectively, compared to the vehicle-treated group $(*, \mathrm{p}<0.05)$. The reduction of infarct volume was dosedependent (\#, $\mathrm{p}<0.05$ ) (Figure 2A). Furthermore, 7 days after HI, whole brains were fixed, imaged, and then sectioned for Nissl staining. Carvacrol pre-treatment $(50 \mathrm{mg} /$ $\mathrm{kg}$ ) also resulted in significantly less brain damage (both in whole brains and coronal sections, $\mathrm{n}=15$ pups) 7 days after HI (Figure 2B) compared to vehicle treatment (with larger brain tissue loss, $\mathrm{n}=12$ pups). Brain imaging at 7 days further supported TTC staining at one day with respect to the neuroprotective effects of carvacrol in HI.

\section{Carvacrol pre-treatment improves neurobehavioral performance after $\mathrm{HI}$}

We further evaluated neurobehavioral outcomes in sham ( $\mathrm{n}=7$ pups), carvacrol-treated ( $\mathrm{n}=15$ pups) and vehicletreated animals ( $\mathrm{n}=12$ pups). Neurobehavioral tests evaluating the geotaxis reflex, cliff avoidance and grip 

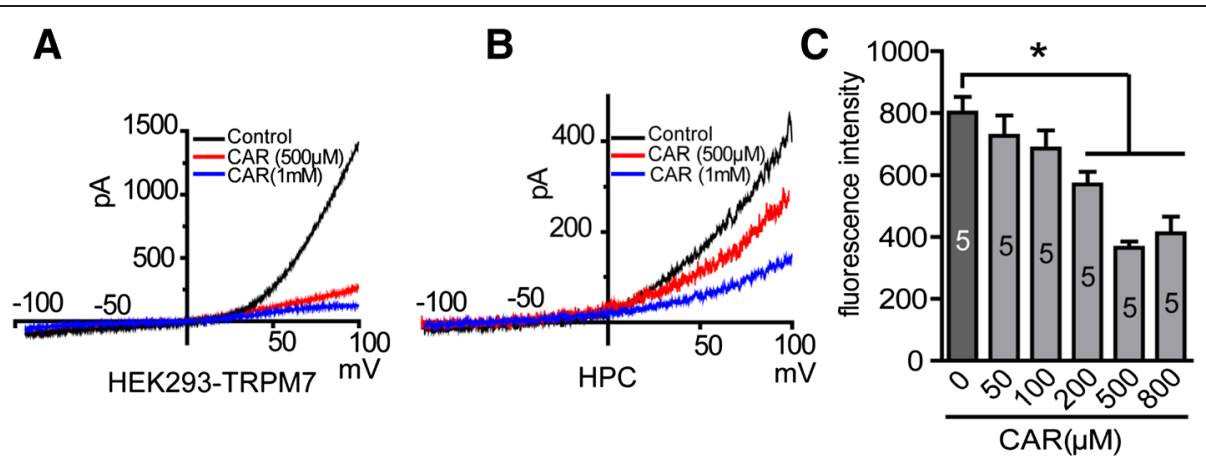

D
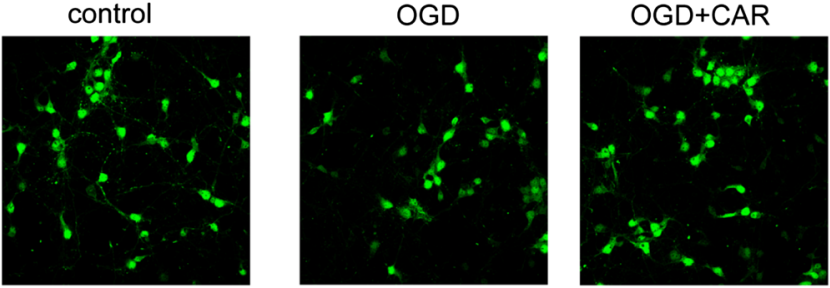

$\mathrm{PI}$
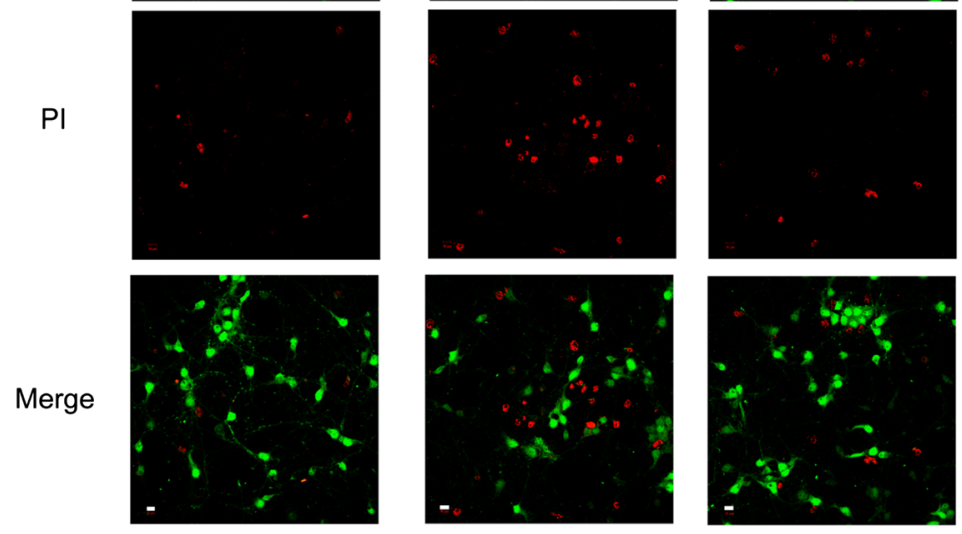

E

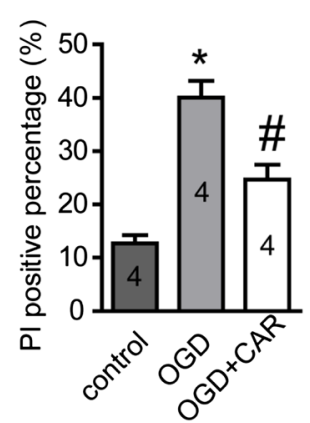

Figure 1 Carvacrol (CAR) inhibits TRPM7 and TRPM7-like currents and protects cortical neurons from OGD-induced injury. A, HEK293 cells over-expressing TRPM7 were induced by tetracycline $(1 \mu \mathrm{M})$ for 24 hours. TRPM7 current was recorded as described in methods section. Representative I-V curves are shown. Perfusion with carvacrol (500 $\mu \mathrm{M}$ and $1 \mathrm{mM}$ ) caused a dramatic decrease in the TRPM7 current in dosedependent manner ( $n=6$ cells). B, TRPM7-like current in primary hippocampal neurons (HPC) was recorded as described in methods section. Perfusion with carvacrol (500 $\mu \mathrm{M}$ and $1 \mathrm{mM}$ ) dose-dependently blocked TRPM7-like current in HPC. Representative I-V curves are shown ( $n=6$ cells). C, cortical neurons were incubated with carvacrol or vehicle (0.1\% DMSO) for 30 min and then treated with OGD for 1 hour and transferred to regular medium for 24 hours. Cells were then stained with PI and the fluorescent intensity was measured using Synergy HT Multi-Mode Micro plate Reader. Results demonstrated that carvacrol $(200-800 \mu \mathrm{M})$ significantly protected neurons from OGD-induced injury ${ }^{*}$, $\mathrm{p}<0.05$ compared with vehicle treated group, $n=5$, One-way ANOVA followed by Newman-Keuls test). $\mathbf{D}$ and $\mathbf{E}$, cortical neurons were treated with carvacrol (300 $\mu \mathrm{M})$ for $30 \mathrm{~min}$, and then OGD and PI staining were conducted as described above. Representative images were taken using a Zeiss LSM 710 Confocal Microscope. Scale bar $=10 \mu \mathrm{m}$. *, $\mathrm{p}<0.05$ compared with control group; \#, $\mathrm{p}<0.05$ compared with OGD group, $\mathrm{n}=4$, One-way ANOVA followed by Newman-Keuls test. 

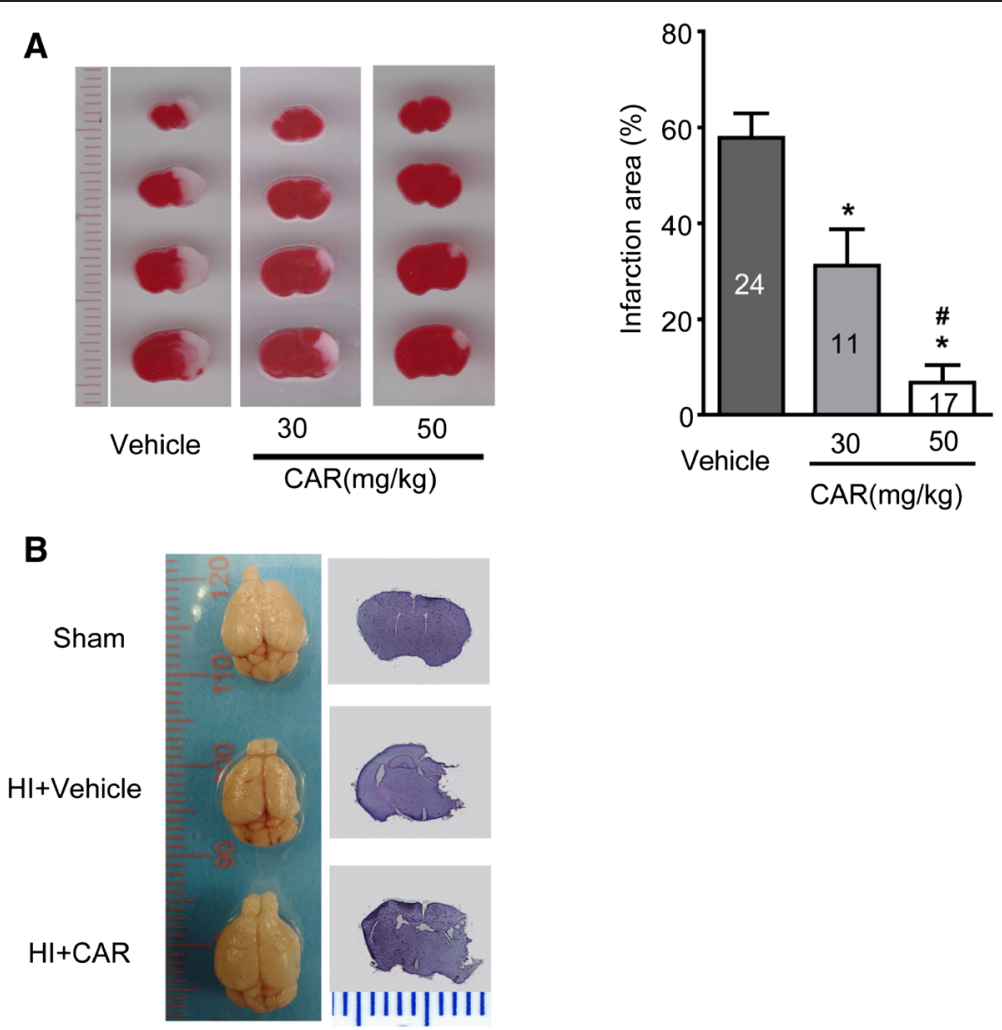

Figure 2 Carvacrol (CAR) pre-treatment reduced infarct volume of neonatal hypoxic-ischemic brain injury in a dose-dependent manner. A, TTC staining was performed as described in methods section. The representative images of TTC-stained coronal brain slices are shown (Left panel). Infarct volume is smaller in carvacrol pre-treatment group than vehicle group ${ }^{*}, p<0.05$ compared with vehicle group. \#, $p<0.05$ compared with carvacrol $30 \mathrm{mg} / \mathrm{kg}$ group, One-way ANOVA followed by Newman-Keuls test). B, representative images of whole brain and Nissl staining for brain slices (sham, $n=7$; vehicle group, $n=12$; carvacrol pretreatment group, $n=15$ ).

were performed 1,3 , and 7 days after $\mathrm{HI}$ in the three groups. Compared with pups in the sham group, neurobehavioral functions of pups in the vehicle-treated $\mathrm{HI}$ group were significantly impaired 1, 3 and 7 days after HI (Figure 3, * $\mathrm{p}<0.05$ ). Geotaxis test performance was significantly better in the carvacrol-treated group 7 days after HI (Figure 3A) compared to the vehicle-treated group ( $3.11 \pm 0.53 \mathrm{~s}$ in carvacrol-treated HI group versus $4.89 \pm 0.67 \mathrm{~s}$ in the vehicle-treated HI group; $\mathrm{p}<0.05$ ). Cliff avoidance test performance was also significantly better in the carvacrol-treated group 3 and 7 days after HI (Figure 3B) compared to the vehicle-treated group $(1.48 \pm 0.14 \mathrm{~s}$ in the carvacrol-treated group versus $1.90 \pm 0.13 \mathrm{~s}$ in the vehicle-treated group 3 days after $\mathrm{HI} ; 1.42 \pm 0.13 \mathrm{~s}$ in the carvacrol-treated group versus $2.01 \pm 0.23 \mathrm{~s}$ in the vehicle-treated group 7 days after HI; $\mathrm{p}<0.05)$. Grip test performance was also significantly better 1, 3 and 7 days after $\mathrm{HI}$ in the carvacroltreated group compared to the vehicle-treated group (Figure $3 \mathrm{C})(\mathrm{p}<0.05)$. Therefore, carvacrol not only reduced brain damage but also improved behavioral outcomes after HI challenge.

\section{TRPM7 protein expression increases in the $\mathrm{HI}$ mouse} brain

We also determined whether TRPM7 expression in the brain changes after HI. Western blotting experiments were carried out to compare TRPM7 protein expression between the ipsilateral and contralateral brain hemispheres 24 hours after HI. We showed that TRPM7 protein expression level was approximately 5 -fold higher in the ipsilateral hemisphere than in the contralateral hemisphere (ipsilateral: $0.42 \pm 0.065$ arbitrary units (AUs) versus contralateral: $0.077 \pm 0.047$ AUs; $p<0.05$, $\mathrm{n}=4$ ) (Figure 4A). This indicates that the TRPM7 channel may be involved in neonatal hypoxic-ischemic brain injury.

\section{Carvacrol pre-treatment protects brain tissue in $\mathrm{HI}$ model from apoptosis}

Apoptosis is involved in neonatal HI-induced neuronal death, which may take time to develop $[16,17]$. It is more common in the immature brain than in the adult brain [18]. Preventing or reducing apoptosis is a therapeutic strategy for neonatal stroke. We examined whether the 
A

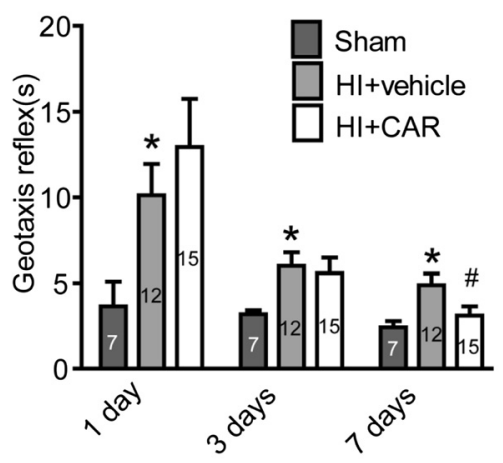

C

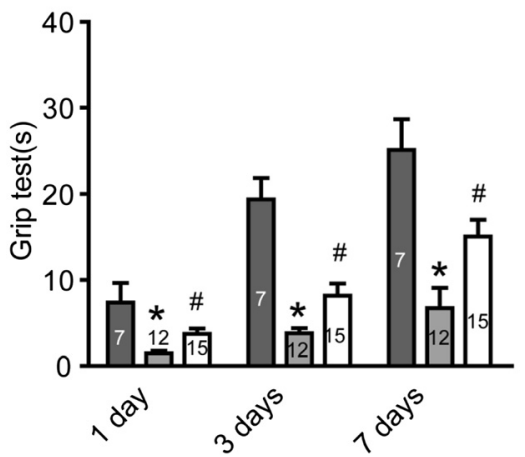

B

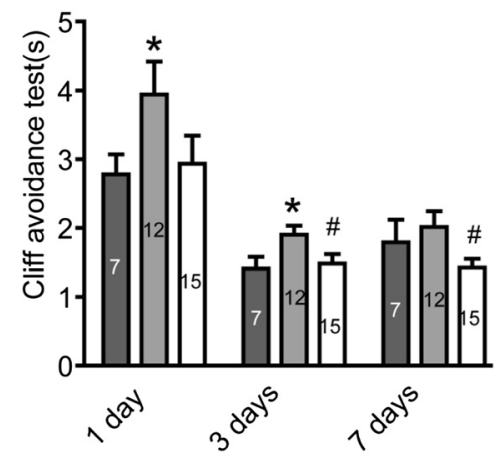

Figure 3 Carvacrol pretreatment improves neurobehavioral performance after cerebral HI. Neurobehavioral evaluation was performed as described in Material and Methods. Geotaxis reflex $\mathbf{( A )}$, cliff avoidance test $\mathbf{( B )}$ and grip test $\mathbf{( C )}$ of sham $(n=7)$, vehicle group $(H \mathrm{H}+$ vehicle, $n=12)$ and carvacrol $(50 \mathrm{mg} / \mathrm{kg})$ pretreatment group $(\mathrm{HI}+\mathrm{CAR}, \mathrm{n}=15)$ were measured 1 day, 3 days and 7 days after $\mathrm{HI}\left({ }^{*}, \mathrm{p}<0.05\right.$ versus sham group; \#, $\mathrm{p}<0.05$ versus vehicle group, One-way ANOVA followed by Newman-Keuls test).

protective effect of carvacrol is associated with the reduction of apoptosis using a TUNEL assay and western blot (cleaved caspase-3, see below). The number of TUNELpositive cells in the vehicle-treated HI group increased significantly with respect to the sham group $(146.33 \pm 16.06$ cells in the vehicle-treated group versus $1.67 \pm 0.33$ cells in the sham group; $\mathrm{p}<0.05, \mathrm{n}=3$ ) (Figure $4 \mathrm{~B}$ and $\mathrm{C}$ ). Carvacrol $(50 \mathrm{mg} / \mathrm{kg}$ ) pre-treatment significantly decreased TUNEL-positive cell counts compared to the vehicle-treated HI group (reduced to $15.00 \pm 1.46$ cells, $\mathrm{p}<0.05, \mathrm{n}=3$ ). Immunofluorescence showed that overexpression of TRPM7 (blue) was accompanied by increasing expression of cleaved caspase-3 (red) in penumbra of the ipsilateral hemisphere, when compared to a similar region on the contralateral hemisphere of the HI mouse (Figure 5). Anti-NeuN antibody was used to label surviving neurons and it showed that there were more degenerating cells and fewer surviving cells in the penumbra of ipsilateral hemisphere compared with a similar region on contralateral side, which shows normal neuron morphology and mostly surviving cells. These results indicate that apoptotic cells in the penumbra of the ipsilateral hemisphere express higher levels of TRPM7 protein, and inhibition of TRPM7 by carvacrol protects cells from apoptosis in neonatal brains of HI mice. Next we investigated if this reduction of TUNEL-positive cells is mediated by any known apoptotic signals.

\section{Carvacrol pre-treatment increases $\mathrm{Bcl}-2 / \mathrm{Bax}$ protein ratio and reduces caspase- 3 cleavage}

TUNEL results indicate that carvacrol may reduce apoptosis in neonatal HI brain injury. We further determined which apoptosis-related proteins may be involved in this effect. Caspase- 3 cleavage has been reported as a major cause of brain injury after neonatal stroke [19]. Bcl-2/ Bax protein ratio is important in the mitochondrial apoptosis pathway [20]. Bcl-2 and Bax protein levels play a key role in brain injury in the neonatal $\mathrm{HI}$ model $[21,22]$. In accordance with other studies [13,19], we found that cleaved caspase- 3 protein expression was significantly greater 24 hours after $\mathrm{HI}$ (normalized to $\beta$-actin, $1.03 \pm 0.08 \mathrm{AUs}$ in the vehicle-treated HI group versus $0.088 \pm 0.003$ AUs in the sham group; $\mathrm{p}<0.05$, $\mathrm{n}=3$ ) (Figure 6A and C). Carvacrol pre-treatment significantly reduced cleaved caspase- 3 levels to $0.75 \pm$ 0.04 AUs ( $p<0.05$ when compared to the vehicle-treated group, $\mathrm{n}=3$ ). We also found that the protein ratio of $\mathrm{Bcl}-$ $2 /$ Bax was significantly lower in the vehicle-treated group 

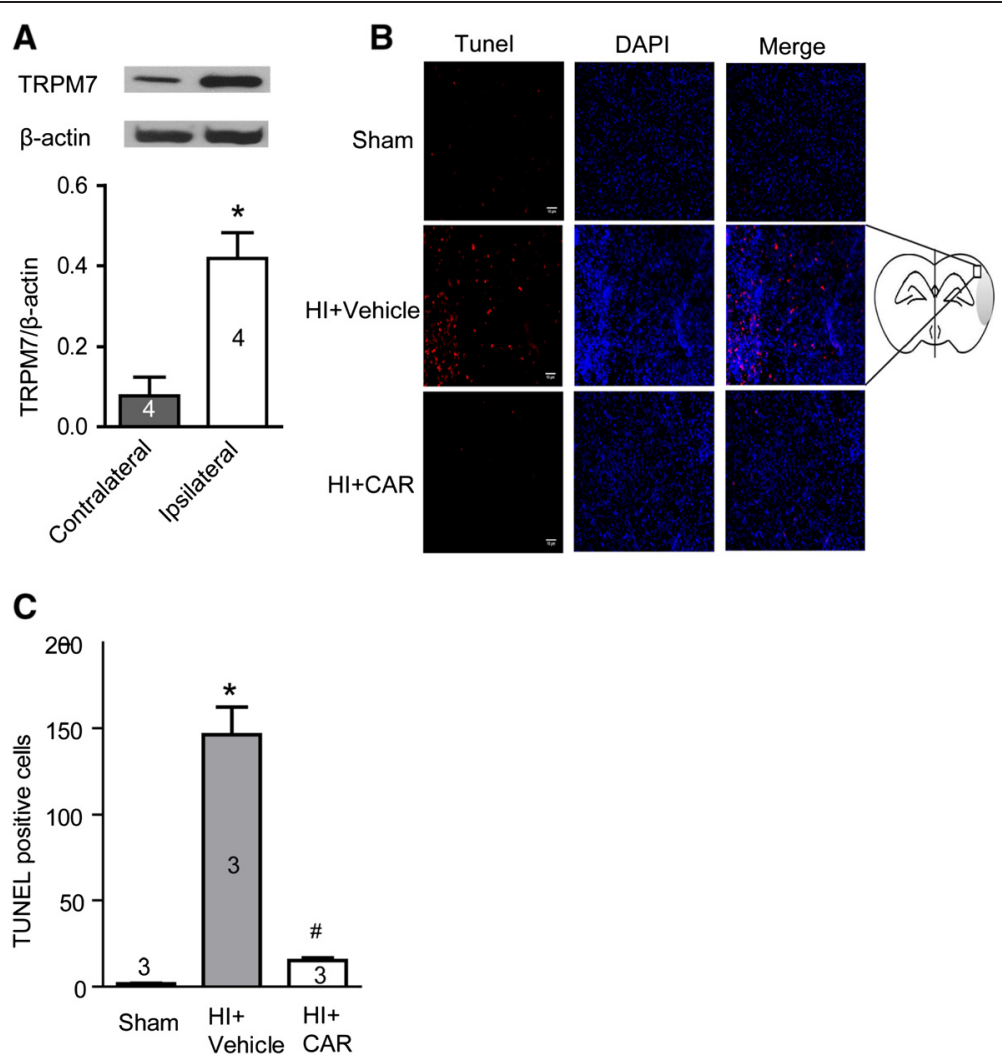

Figure 4 TRPM7 protein expression in the cortex of $\mathrm{HI}$ mice and TUNEL staining of mouse brain slices. A, western blotting results of TRPM7 protein expression in the injured hemisphere (ipsilateral) and uninjured contralateral hemisphere 24 hours after HI. TRPM7 protein expression level is higher in injured hemisphere than the contralateral hemisphere $\left.{ }^{*}, p<0.05, n=4\right)$. B, TUNEL staining was carried out 3 days after HI and images were viewed using fluorescence microscopy. Left panel shows the representative images of TUNEL staining. Red fluorescence in TUNEL-positive cells indicates apoptosis. Blue fluorescence is DAPI staining for nucleus. Scale bar represents $10 \mu \mathrm{m}$. Right panel shows the mouse brain coronal sections. C, Quantitative analysis of TUNEL-positive cells showed that pre-treatment with carvacrol (50 mg/kg) reduced the number of TUNEL positive cells $\left({ }^{*}, p<0.05\right.$ compared with sham group. \#, $p<0.05$ compared with vehicle group, $n=3$, One-way ANOVA followed by Newman-Keuls test).

compared to the sham group $(0.038 \pm 0.002$ AUs in vehicle-treated group versus $0.067 \pm 0.006 \mathrm{AUs}$ in sham group; $\mathrm{p}<0.05, \mathrm{n}=3$ ) (Figure $6 \mathrm{~A}$ and $\mathrm{B}$ ); while carvacrol pre-treatment $(50 \mathrm{mg} / \mathrm{kg})$ restored $\mathrm{Bcl}-2 / \mathrm{Bax}$ ratio to $0.055 \pm 0.004$ AUs ( $<0.05$ to vehicle-treated group, $n=3$ ). Therefore, carvacrol is able to inhibit pro-apoptotic signaling pathways in neonatal hypoxic-ischemic brain injury. Next, the effects of carvacrol on Bcl-2/Bax ratio were measured in an OGD-induced cortical neuron injury model. As shown in Figure 6E and $\mathrm{F}$, cortical neurons exposed to OGD had a lower Bcl-2/Bax ratio, while the Bcl-2/Bax ratio increased in the carvacrol $(300 \mu \mathrm{M})$ pre-treatment group (*, \#, $\mathrm{p}<0.05, \mathrm{n}=4)$.

\section{Carvacrol pre-treatment increases phosphorylated Akt (p-Akt) level after HI brain injury}

Activation of the PI3K/Akt pathway is a protective signaling pathway involved in neuroprotection in stroke [23]. Carvacrol has been reported to increase Akt phosphorylation in a focal ischemia model [13]. In this study, we found that $\mathrm{p}$-Akt/t-Akt was significantly lower in the vehicle-treated HI group $(0.26 \pm 0.02$ AUs in the vehicletreated group versus $0.53 \pm 0.02$ AUs in sham group; $\mathrm{p}<$ $0.05, \mathrm{n}=3$ ); while carvacrol pre-treatment restored the $\mathrm{p}$-Akt/t-Akt ratio to $0.50 \pm 0.1 \mathrm{AU}$ ( $\mathrm{p}<0.05$ with respect to the vehicle-treated group, $n=3$ ) (Figure 6A and D). Therefore, carvacrol is able to promote pro-survival signaling in neonatal hypoxic-ischemic brain injury.

\section{Discussion}

In this study, we show that: 1) carvacrol inhibits TRPM7 and TRPM7-like currents in TRPM7 over-expressing HEK293 cells and mouse hippocampal neurons, respectively; 2) TRPM7 protein expression in the brain increases 24 hours after $\mathrm{HI}$; 3) pre-treatment with the TRPM7 channel blocker carvacrol is neuroprotective and improves behavioral outcomes in a mouse model of neonatal hypoxic-ischemic brain injury; 4) carvacrol inhibits pro-apoptotic signals in $\mathrm{HI}$ by increasing Bcl-2/ Bax protein ratio and reducing caspase- 3 activation; and 5) carvacrol promotes pro-survival signals in HI by restoring the PI3K/Akt signaling pathway. These findings 


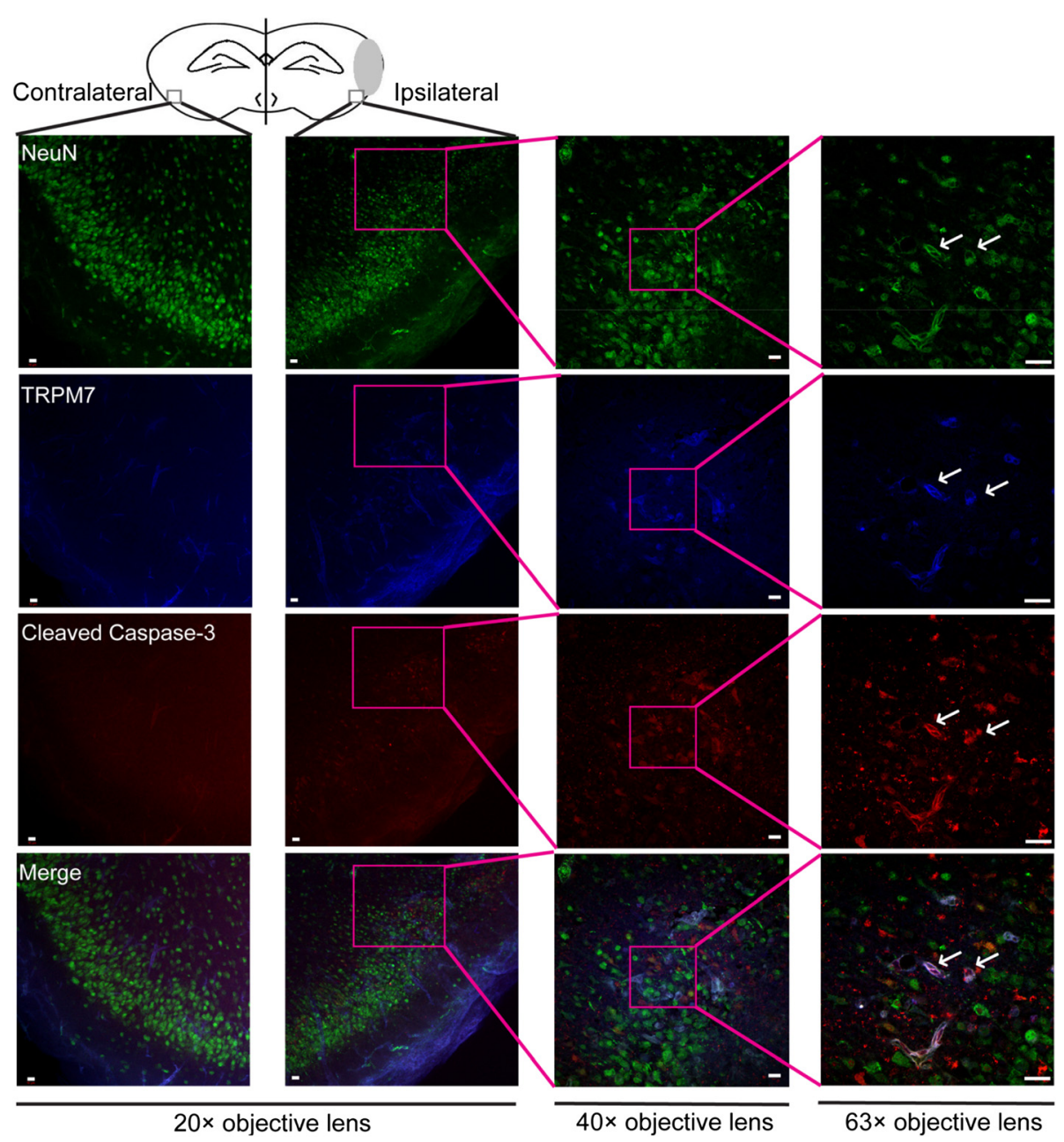

Figure 5 Immunofluorescence assay of TRPM7 and cleaved caspase- 3 expression in brain sections of HI mice. Triple immunofluorescence staining was employed for TRPM7, cleaved caspase-3 and NeuN in the penumbra of the injured hemisphere (ipsilateral) and in a similar region of the uninjured contralateral hemisphere 24 hours after HI. Representative images are shown. Green, blue and red fluorescence indicates NeuN, TRPM7 and cleaved caspase-3, respectively. The white arrow indicates the co-localization of TRPM7 and activated caspase-3 with NeuN. N =3, Scale bar in 20x and 40x magnifying images $=10 \mu \mathrm{m}$. Scale bar in 63x magnifying images $=20 \mu \mathrm{m}$.

suggest that carvacrol has therapeutic potential in preventing and treating neonatal hypoxic-ischemic brain injury.

First, we employed whole-cell patch clamp to confirm that carvacrol inhibited TRPM7 current in HEK293 cells over-expressing TRPM7 and in cultured hippocampal neurons (Figure 1). Our results show that carvacrol inhibits TRPM7 currents in HEK293 cells and TRPM7like currents in cultured neurons in a dose-dependent manner. This is consistent with other studies [12]. Next, we detected neuroprotective effects of carvacrol in vitro using an OGD model in cultured neurons. We showed that carvacrol inhibits OGD-induced neuronal cell death in vitro in a dose-dependent manner. As OGD is a wellaccepted in vitro hypoxia-ischemia cell model for drug testing [24], our findings indicated that carvacrol may also protect neurons from hypoxia-ischemia brain injury in vivo. Hence, we further evaluated the effects of carvacrol in vivo using a neonatal stroke model of HI.

The neonatal hypoxic-ischemic brain injury model used in this study was adapted from the method described by Rice et al. [25], with minor modifications. It is a widely used neonatal stroke model, and has been employed to explore drug intervention targets for neonatal hypoxic-ischemic brain injury [26,27]. We have established this HI model to study the neuroprotective effects of a volume-regulated anion channel blocker [28] and hypoxic preconditioning [29]. In addition to the OGD in vitro model, we also evaluated the effects of carvacrol in an in vivo neonatal stroke model.

Upregulation of TRPM7 protein levels has been reported to occur 20 and 24 hours after MCAO and ischemia-reperfusion in adult rats [7,9]. However, brain TRPM7 protein expression after neonatal hypoxic- 
A

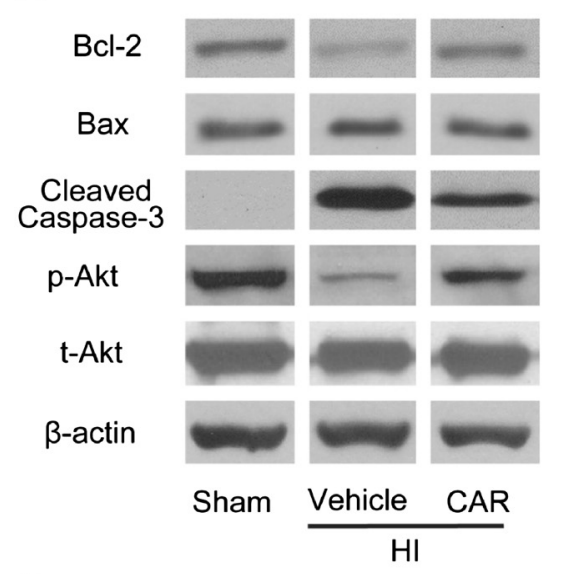

C

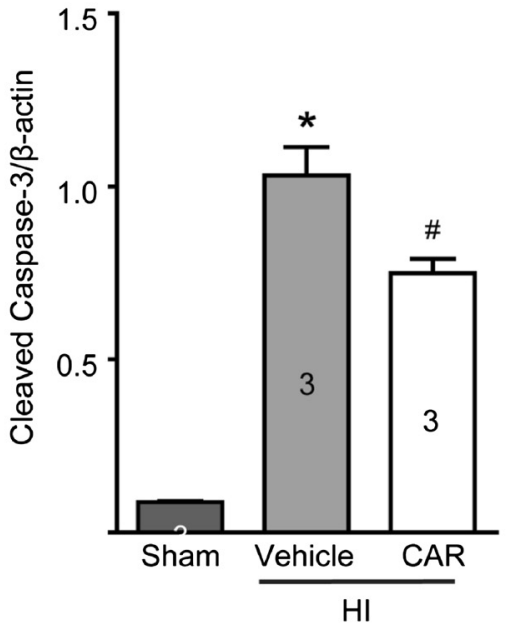

E

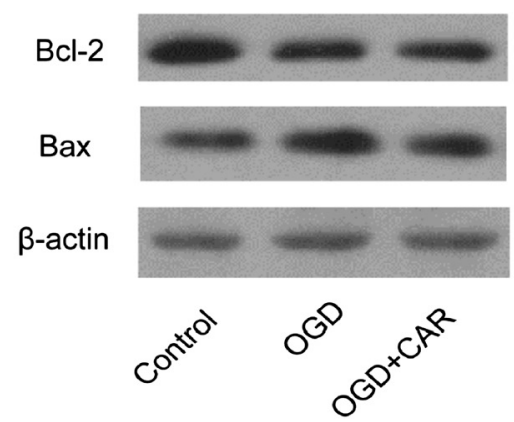

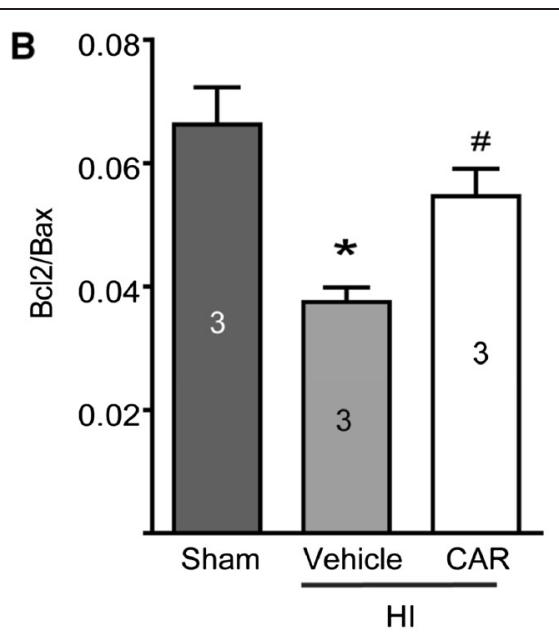

D

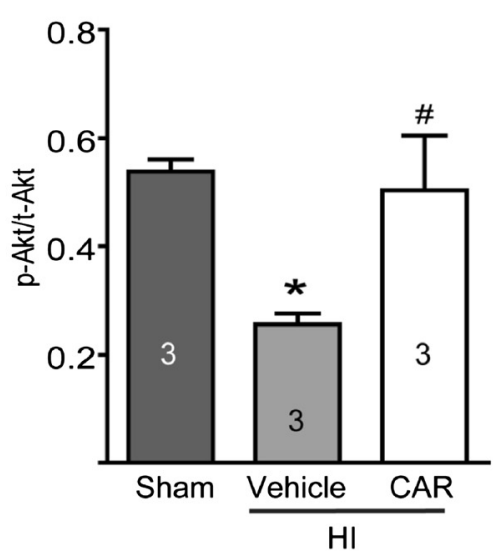

$\mathbf{F}$

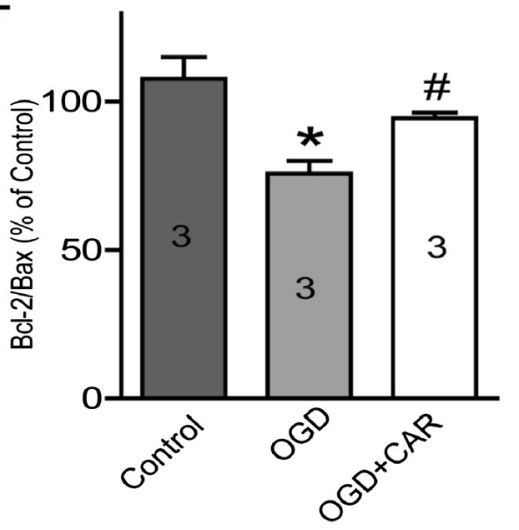

Figure 6 Western blotting results. Experiments were carried out as described in the methods section. A, representative images of $\mathrm{BCl}-2$ (26 kDa), Bax (20 kDa), cleaved caspase-3 (19/17 kDa), p-Akt (Ser 473, $60 \mathrm{kDa}$ ) and t-Akt (60 kDa) protein expression. $\beta$-actin (42 kDa) was used as a loading control. Carvacrol $(50 \mathrm{mg} / \mathrm{kg}$ ) pretreatment increased the protein ratio of $\mathrm{BCl}-2 / \mathrm{Bax}$ (B), decreased cleaved caspase-3 protein expression (C) and increased $p$-Akt/t-Akt protein ratio (D) 24 hours after $\mathrm{HI}(*, p<0.05$ versus sham group; $\#, p<0.05$ versus vehicle group, $n=3$, One-way ANOVA followed by Newman-Keuls test). When primary mouse cortical neurons were exposed to OGD for 60 minutes and additional 18 hours in normal culture medium, BCl-2/Bax ratio in the OGD group was lower than that of the control group, and significantly increased in the Carvacrol $\left(300 \mu \mathrm{M}\right.$ ) pre-treatment group ( $\mathbf{E}$ and $\mathbf{F},{ }^{*}, p<0.05$ versus the sham group; $\#, p<0.05$ versus the vehicle group, $n=3$, One-way ANOVA followed by Newman-Keuls test). 
ischemic brain injury was previously unknown. Here we compared TRPM7 protein expression between ipsilateral and contralateral brain hemispheres from neonatal mouse pups subjected to HI 24 hours prior. Upregulation of TRPM7 protein expression occurred in apoptotic cells in ipsilateral brain tissue. This suggests that TRPM7 is relevant to HI-induced brain injury and can potentially serve as a therapeutic target for hypoxicischemic brain injury, the function of which can be modulated by carvacrol.

In this study, carvacrol was intraperitoneally injected 30 minutes before pups were subjected to hypoxicischemic injury. Carvacrol is a lipophilic compound with a small molecular weight (mol wt, 150.22) [30]. Carvacrol likely crosses the blood-brain barrier (BBB) in neonatal mice because administering carvacrol intraperitoneally at $2 \mathrm{~h}$ after reperfusion showed the neuroprotective effects on brain injury in an adult mouse MCAO model [13]. The $\mathrm{BBB}$ of newborns is considered to be more permeable than that of adults for certain compounds [31]. Furthermore, increased BBB permeability often results from hypoxia-ischemia [32]. These factors, combined with the physicochemical characteristics of carvacrol, suggest that carvacrol crosses the BBB of HI mice. We showed that carvacrol pre-treatment reduced brain damage 1 day after HI as assessed by TTC stain and also 7 days after HI using whole brain imaging and Nissl stain. The neuroprotective effects of carvacrol are dose-dependent. Neonatal hypoxic-ischemic brain injury leads to motor and cognitive deficits, and seizures [33]. Therefore, we also evaluated the neurobehavioral function of carvacrol-treated mice compared to vehicle-treated $\mathrm{HI}$ and sham mice. Carvacrol improved behavioral outcomes in a battery of tests including geotaxis reflex, cliff avoidance and grip tests. Therefore, our results indicate that carvacrol not only reduced infarct volume, but also improved the return of neurobehavioral function. It was reported that carvacrol improved diabetes-related cognitive deficits in a Morris water maze test in rats [34]. The effects of carvacrol on HI-induced cognitive dysfunction remain unknown. Further experiments are required to evaluate its effects over the long term.

Apoptosis is considered to be a key pathological trigger of delayed neuronal death following transient global ischemia [16,17]. Apoptosis is more common in the immature brain than adult brain [18]. Our study detected the apoptosis in neonatal hypoxic-ischemic brain injury first using TUNEL assay followed by western blot for apoptosis-related proteins. The number of TUNELpositive cells in the carvacrol-treated group was significantly lower than the number found in the vehicletreated group. This is consistent with other reports showing anti-apoptotic effects of carvacrol in myocardial cells [35]. The mitochondria-dependent pathway for apoptosis is regulated by the $\mathrm{Bcl}-2$ family of proteins, each of which either promotes or prevents apoptosis. $\mathrm{Bcl}-2$ is involved in HI brain apoptosis [36]. The total expression of Bcl-2 decreases 24 hours after neonatal HI in vivo [36], while Bax expression increases. Enhancing Bcl-2 or reducing Bax expression provides protection from injury in neonatal HI brains [36-38]. The ratio of Bcl-2/Bax protein affects mitochondrial membrane permeability and regulates mitochondrially-mediated apoptosis pathways. In our study, western blotting revealed that the ratio of $\mathrm{Bcl}-2 / \mathrm{Bax}$ decreased in neonatal $\mathrm{HI}$ pups. With carvacrol pre-treatment, the protein ratio of Bcl-2/Bax was greater. In addition, carvacrol also inhibited an OGD-induced decrease in Bcl-2/Bax, suggesting that $\mathrm{Bcl}-2 / \mathrm{Bax}$ ratio in the mitochondrial apoptotic signaling pathway is involved in the anti-apoptotic effects of carvacrol. It is reported that $\mathrm{Bcl}-2$ reduction in neonatal $\mathrm{HI}$ can be prevented by the calpain inhibitor CX295 [36]. M-calpain is an enzyme substrate of TRPM7 [39]. It suggests that carvacrol blocks TRPM7 function partly by reducing its enzyme substrate, $\mathrm{m}$ calpain, to restore $\mathrm{Bcl}-2$ expression leading to prevention of apoptosis in neonatal hypoxic-ischemic brain injury. Further research is required to reveal this mechanism. Caspase-3, which is the protein downstream of mitochondrial and death receptors in the apoptotic signaling pathway, has been identified as a key mediator of apoptosis in animal models of ischemic stroke [40]. Inhibition of caspase- 3 activation is an established therapeutic strategy for neonatal $\mathrm{HI}$ brain injury $[41,42]$. In an adult MCAO mouse model, it was reported that carvacrol treatment decreased cleaved caspase-3 protein level after cerebral ischemia injury [13]. We also found that cleaved caspase- 3 increased in HI pups 24 hours after hypoxiaischemia. Carvacrol pre-treatment significantly attenuated the level of cleaved caspase-3. Hence, carvacrol effectively prevents apoptosis in neonatal hypoxic-ischemic brain injury, likely through the mitochondrial $\mathrm{Bcl}-2 / \mathrm{Bax} /$ caspase- 3 signaling pathway.

Activation of PI3K/Akt, a critical pro-survival signaling pathway, plays a protective role in cerebral ischemia injury [23]. It has been reported that the phosphorylation level of Akt (p-Akt) is reduced 8 and 24 hours after HI in rats [43]. In our study, p-Akt protein expression was reduced 24 hours after HI, which is consistent with previous results [43]. Carvacrol pre-treatment significantly restored $\mathrm{p}$-Akt protein expression. Thus, the PI3K/Akt signaling pathway also contributes to the protective effects of carvacrol in neonatal stroke.

This study's use of a pre-treatment paradigm provides "Proof of Principle" for: 1) the involvement of a nonglutamatergic mechanism (TRPM7) in HI brain injury, and 2) the idea that TRPM7 blockers (i.e. carvacrol) are a promising target for drug development against HI- 
induced brain injury. Clinically, acute ischemic stroke patients are given therapies after symptom onset. It has been reported that post-stroke treatment with carvacrol also inhibited brain injury in an adult mouse model of middle cerebral artery occlusion model, with a therapeutic window of up to 6 hours [13], suggesting that carvacrol has potential as a stroke treatment. Future study will test different time courses- including posttreatment- for carvacrol in the neonatal mouse $\mathrm{HI}$ model and evaluate its therapeutic widow for neonatal stroke. In conclusion, carvacrol pre-treatment protects brains from hypoxic-ischemic injury, improves neurobehavioral outcomes, promotes pro-survival and inhibits pro-apoptosis signaling pathways in a mouse model of neonatal hypoxic-ischemic brain injury. Neuroprotection is likely mediated by the inhibition of TRPM7 current and the resultant up-regulation of $\mathrm{Bcl}-2 / \mathrm{Bax}$ and $\mathrm{p}$-Akt to decrease caspase- 3 activation. Since carvacrol is considered a safe substance and is widely used as a food additive $[44,45]$, it can potentially be used as a bioactive molecule for the prevention and treatment of neonatal hypoxic-ischemic brain injury and its related brain disorders.

\section{Materials and methods \\ Animals}

All protocols were carried out in accordance with Canadian Council on Animal Care guidelines and approved by the local Animal Care Committee (Office of Research Ethics, University of Toronto). Timed-pregnant CD1 mice were purchased from Charles River Laboratories (Sherbrooke, Quebec, Canada) and gave birth in the animal facility at the University of Toronto. Mice were housed with an ambient temperature of $20 \pm 1^{\circ} \mathrm{C}$ and a 12-hour light/dark cycle with free access to a standard laboratory chow diet and water. All experiments were performed in a blinded manner; experimenters were not aware of treatment information for all assessments.

\section{Materials and drugs}

Carvacrol (cat\#W224502), Dimethyl sulfoxide (DMSO), Tetrodotoxin (TTX), DL-2-Amino-5-phosphonopentanoic acid (APV), CNQX, nimodipine, poly-D-lysine (Sigma) and tetracycline were purchased from SigmaAldrich, USA. MEM medium, fetal bovine serum (FBS) and other cell culture materials were purchased from Gibco Life Technologies Corporation (USA). All other reagents used were purchased from Sigma-Aldrich, USA unless stated otherwise.

\section{Cell cultures}

HEK293 cells with stable expression of tetracyclineinducible flag-murine TRPM7/pCDNA4 were cultured with Minimum Essential Medium (MEM) supplemented with 10\% FBS, glutamax-1 (2 mM, Invitrogen, USA), blasticidin $(5 \mu \mathrm{g} / \mathrm{mL})$ and zeocin $(0.4 \mathrm{mg} / \mathrm{mL}$, Invitrogen, USA).

Primary neuronal culture was performed using E16 CD1 mice as described previously [11]. In brief, hippocampi or cortices were dissected and digested with $0.025 \%$ Trypsin/ EDTA at $37^{\circ} \mathrm{C}$ for $15 \mathrm{~min}$. Cell density was calculated using an Improved Neubauer hemocytometer, and then plated on poly-D-lysine-coated glass $12 \mathrm{~mm}$ round coverslips (German Glass, Bellco cat \#1943-10012) for electrophysiology experiments, or a poly-D-lysine-coated 96-well plate for OGD experiments. The cells were kept in $5 \% \mathrm{CO}_{2}$ and 95\% humidified air at $37^{\circ} \mathrm{C}$ in serum-free culture medium (Neurobasal medium supplemented with $1.8 \%$ B-27, 2\% HEPES, $0.25 \%$ Glutamax, and 1\% antibiotic-antimyocotic).

\section{Electrophysiology recordings}

TRPM7 and TRPM7-like currents were recorded by whole-cell patch-clamp using $400 \mathrm{~ms}$ voltage ramps from -100 to $+100 \mathrm{mV}$, with an interval of $5 \mathrm{~s}$ at $2 \mathrm{kHz}$ and digitized at $5 \mathrm{kHz}$ using MultiClamp 700B, Digidata 1322A and Clampex 9.2 software. The pipette solution for hippocampal neurons contained (in mM): $140 \mathrm{CsF}$, $35 \mathrm{CsOH}, 10 \mathrm{HEPES}, 2$ tetraethylammonium chloride, 11 EGTA, $1 \mathrm{CaCl}_{2}, 2 \mathrm{MgCl}_{2}$ and $2 \mathrm{~K}_{2} \mathrm{ATP}$ (pH 7.3, 290$300 \mathrm{mOsm} / \mathrm{L}$ ) [46]. The pipette solution for recordings in HEK293 cells contained (in mM): 145 cesium methanesulfonate, 10 EGTA, $8 \mathrm{NaCl}$ and 10 HEPES, pH adjusted to 7.2 with $\mathrm{CsOH}$. The extracellular solution for whole-cell patch-clamp recordings contained $140 \mathrm{~mm}$ $\mathrm{NaCl}, 5 \mathrm{~mm} \mathrm{KCl}, 2 \mathrm{~mm} \mathrm{CaCl}_{2}, 20 \mathrm{~mm} \mathrm{HEPES}$, and $10 \mathrm{~mm}$ glucose, $\mathrm{pH}$ adjusted to $7.4(\mathrm{NaOH})$ [46]. Patch pipette resistance was between 3-5 megaohms. When recording the TRPM7-like currents in hippocampal neurons, 500nM TTX, $25 \mu \mathrm{M}$ APV, $40 \mu \mathrm{M}$ CNQX, and $5 \mu \mathrm{M}$ nimodipine was added to the bath solution.

\section{OGD experiment}

Oxygen and glucose deprivation (OGD) has been well established in our lab [28]. In brief, cortical neurons cultured in 96-well clear plates were incubated with carvacrol in oxygen-deprived solution for 30 minutes, followed by incubation in an anaerobic chamber flushed with $5 \% \mathrm{CO}_{2}$ and $95 \% \mathrm{~N}_{2}(\mathrm{v} / \mathrm{v})$ at $37^{\circ} \mathrm{C}$ for 60 minutes. Cells were then incubated in normoxic conditions for an additional 24 hours.

\section{PI staining}

OGD-induced cell injury was determined by propidium iodide (PI) staining using a microplate reader (Syngery $\mathrm{H} 1$, Biotek, USA) to detect fluorescence intensity. In brief, cells were incubated with PI $(5 \mu \mathrm{g} / \mathrm{ml}$, Molecular Probes, USA) for 20 minutes. Fluorescence intensity was read using an excitation wavelength of $488 \mathrm{~nm}$, and an 
emission wavelength of $630 \mathrm{~nm}$. PI enters the cell and stains DNA in the nucleus when cell membrane integrity is compromised during OGD. Greater fluorescence intensity indicates more cell damage in OGD. Moreover, images of PI staining were taken to show damaged cells. In brief, cells were stained with PI for $20 \mathrm{~min}$, and then fixed with $4 \%$ paraformaldehyde in PBS for $20 \mathrm{~min}$ at RT. After washing with PBS 3 times, cells were incubated overnight with anti-NeuN antibody (1:200, Millipore, MAB377) and subsequently incubated with anti-mouse secondary antibody conjugated to Alexa Fluor ${ }^{\bullet} 488$ (1:500, CST 4408) for 2 h at RT. Images were taken using Zeiss LSM 710 Confocal Microscope. The number of cells was counted using Image J. The percentage of PI positive cells $(\%)=($ PI positive cell number/total cell number)*100\%.

\section{Hypoxia-Ischemia and carvacrol administration}

Mouse HI was carried out according to a method described previously $[28,29]$. Briefly, postnatal Day 7 (P7) mice of both genders were anesthetized with isoflurane (3.0\% for induction and $1.5 \%$ for maintenance). The right common carotid artery (CCA) was exposed and ligated. Mice were then returned to their dam and allowed to recover for 1.5 hours and placed in an airtight, transparent chamber (A-Chamber A-15274 with ProOx 110 Oxygen Controller/E-720 Sensor, Biospherix, NY, USA) perfused with humidified gas mixture containing $7.5 \%$ oxygen balanced with $92.5 \%$ nitrogen at $37^{\circ} \mathrm{C}$ for 100 minutes. Chamber temperature was monitored using a homoeothermic blanket control unit (K-017484 Harvard Apparatus, Massachusetts, USA). Sham control animals underwent anesthesia and the common carotid artery was exposed without ligation and hypoxia.

Pups were randomly assigned to one of the following groups: sham control group (Sham), HI + vehicle (Vehicle, $0.5 \%$ DMSO in normal saline) or $\mathrm{HI}+$ carvacrol (CAR, 30 and $50 \mathrm{mg} / \mathrm{Kg}$ ). Carvacrol was dissolved in DMSO and then diluted with normal saline (NS). Vehicle solution was prepared with equivalent DMSO in NS without carvacrol. Equivalent vehicle and carvacrol solution were administered intraperitoneally $30 \mathrm{~min}$ before induction of $\mathrm{HI}$.

\section{Infarct volume evaluation, whole brain imaging and histology}

2, 3, 5-Triphenyltetrazolium chloride (TTC) staining was carried out as described previously [28,29]. Briefly, 24 hours after HI, mice were anesthetized with isoflurane and sacrificed. Brains were removed and cut into four coronal sections (approximately $2 \mathrm{~mm}$ apart). Brain slices were immersed in TTC solution (1\% in PBS, protected from light), incubated at $37^{\circ} \mathrm{C}$ for 15 minutes, and scanned with a high-resolution scanner (Canon). The total infarct area of each section was determined by the change in coloration. Infarct size was determined using an image-analysis system (NIH Image J). The following values were measured for each level brain slice: infarction area (A), the area of the ipsilateral hemisphere (B), and the area of the contralateral hemisphere $(\mathrm{C})$. Corrected infarct volume $(\mathrm{CIV}),(\%)=[(\mathrm{B} 1+\mathrm{B} 2+\mathrm{B} 3+\mathrm{B} 4)-$ $(\mathrm{A} 1+\mathrm{A} 2+\mathrm{A} 3+\mathrm{A} 4)] /(\mathrm{C} 1+\mathrm{C} 2+\mathrm{C} 3+\mathrm{C} 4) * 100[29,47]$.

Whole brains were dissected 7 days after $\mathrm{HI}$ and fixed in $4 \%$ paraformaldehyde overnight. Brain tissues also were sectioned coronally for Nissl staining $(0.1 \%$ cresyl violet). Briefly, brain slices of $30 \mu \mathrm{m}$ were stained with $0.1 \%$ cresyl violet for 2 minutes and rinsed quickly in distilled water. Representative images were captured using a camera (Olympus, Japan) in the same field.

\section{Neurobehavioral evaluation}

All neurobehavioral evaluations were performed in a blinded manner. 1, 3 and 7 days after HI, animals were evaluated with 3 neurobehavioral tests [29].

Negative geotaxis is an automatic, stimulus-bound orientation movement, which is considered to be diagnostic of vestibular and/or proprioceptive function. Pups were placed head down in the middle of a plane inclined at an angle of $45^{\circ}$ [48]. The time taken to rotate $180^{\circ}$ was recorded. The latency to make a $180^{\circ}$ turn was recorded up to a maximum time of $60 \mathrm{~s}$.

Cliff avoidance reaction is used to assess the integration of exteroceptive input and locomotor output. Pups were placed on the edge of a platform, and it was determined how much time was required for the pup to remove both paws from the edge, either by backing off or turning away from the cliff. If the pup did not respond within $60 \mathrm{~s}$, it was recorded as $60 \mathrm{~s}$ [49].

A grip test was used to assess force and fatigability. Pups were suspended by their forepaws on a wire stretched horizontally $30 \mathrm{~cm}$ over a cotton pad in a cage. The time it took for the animal to fall was recorded [50].

\section{Immunofluorescence staining}

Brain tissues were fixed in $4 \%$ paraformaldehyde overnight and then sectioned $(35 \mu \mathrm{m})$ using a Vibratome Series 1000. TUNEL labeling was carried out using TUNEL Apoptosis Detection Kit according to the manufacturer's instructions (Millipore, USA, S7165) [29]. Nuclei were counterstained with DAPI. Images were taken using a Zeiss Axioscope microscope. For NeuN, cleaved caspase-3 and TRPM7 triple staining, brain slices were incubated with anti-NeuN (1:200, Millipore, MAB377), anti-cleaved caspase-3 (1:400, Cell Signaling Technology, \#9661) and anti-TRPM7 (1:50, Abcam, ab729) at $4^{\circ} \mathrm{C}$ overnight. Then brain slices were incubated corresponding secondary antibody from Abcam (1:500): Donkey Anti-Mouse IgG H\&L (Alexa Fluor ${ }^{\circledR} 488$ ), Goat Anti- 
Rabbit IgG H\&L (Alexa Fluor ${ }^{\circ}$ 568) and Donkey AntiGoat IgG H\&L (Alexa Fluor ${ }^{\circ}$ 405). Images were taken using Zeiss LSM 710 Confocal Microscope.

\section{Western blotting}

Western blotting experiments were carried out as described previously [51,52]. Pups were sacrificed and brain samples were collected on dry ice 24 hours after HI. Proteins of the ipsilateral hemisphere were extracted by homogenization in RIPA buffer with proteinase inhibitor cocktail (Santa Cruz Biotechnology, USA). Protein concentrations were determined using the bicinchoninic acid (BCA) method (Pierce). Samples were separated on a $10 \%$ SDS-PAGE gel and proteins were then transferred to a nitrocellulose membrane (200 mA per gel, $60 \mathrm{~min})$. Blots were probed with anti-TRPM7 antibody (1:1000, Abcam, ab85016), anti-Bax Antibody (1:1000), anti-Bcl-2 (D17C4) Rabbit mAb (1:1000), anti-phospho-Akt (Ser473, 1:1000), anti-Akt (C67E7, 1:1000) and anti-cleaved caspase-3 (1:500) antibodies (above antibodies were purchased from Cell Signaling Technology, Danvers, Mass). Horseradish peroxidase-conjugated anti-mouse or anti-rabbit IgG antibodies (1:7500, Sigma) were used as secondary antibodies and were detected with the ECL system (PerkinElmer, Inc, USA). $\beta$-actin (1:3000, Sigma) was used as a control for protein loading. Images were analyzed using an image-analysis system (NIH Image J 1.47v). Protein expression was normalized to that of $\beta$-actin and expressed as percentage of control.

\section{Data analysis}

Data is presented as mean \pm SEM. Statistical differences between groups were analyzed using a one-way ANOVA with subsequent Newman-Keuls test for multiple comparisons and Student's $t$-test to compare two groups. $\mathrm{p}<$ 0.05 was considered statistically significant.

\section{Competing interests}

The authors declare that they have no competing interests.

\section{Authors' contributions}

WC, BX, AJX, LL, FXY, RL performed experiments; WC, ZPF and HSS wrote the manuscript; $W C, E T, A B$, and CLFS contributed to manuscript preparation; all authors discussed the results, analyzed data and commented on the manuscript; ZPF and HSS developed the concepts and designed the study. All authors read and approved the final manuscript.

\section{Authors' information}

WC is currently a Postdoctoral Fellow at the University of Toronto, graduated from Sun Yat-Sen University and was a lecturer for Department of Pharmacology, School of Pharmaceutical Sciences, Guangzhou Medical University, China. Current address for XB is Jilin University, China and for AJX is Jiangxi University of Traditional Chinese Medicine, China.

\section{Acknowledgements}

This work was supported by the following grants: Natural Sciences and Engineering Research Council of Canada (NSERC) Discovery Grants to ZPF (RGPIN 249962) and to HSS (RGPIN 402733); Heart and Stroke Foundation of Canada (HSFC) (NA7198 \& G-13-0003069) and The Scottish Rite Charitable Foundation of Canada (\#12104) to HSS; a University of Toronto and
Universidade de Sao Paulo Joint Call Proposal grant to HSS, ZPF and LRGB; China Scholarship Council (CSC) Fellowships to BX, AJX, LL and RL; a Canadian Institute of Health Research (CIHR) Frederick Banting and Charles Best Canada Graduate Scholarship to ET, Ontario Graduate Scholarship to AB (OGS-PhD), and a New Investigator Award from the Heart and Stroke Foundation of Canada (HSFC) to ZPF.

\section{Author details}

'Department of Surgery, Faculty of Medicine, University of Toronto, 1 King's College Circle, Toronto M5S 1A8, Canada. ${ }^{2}$ Department of Physiology, Faculty of Medicine, University of Toronto, 1 King's College Circle, Toronto M5S 1A8, Canada. ${ }^{3}$ Department of Pharmacology, Faculty of Medicine, University of Toronto, 1 King's College Circle, Toronto M5S 1A8, Canada. ${ }^{4}$ Institute of Medical Science, Faculty of Medicine, University of Toronto, 1 King's College Circle, Toronto M5S 1A8, Canada. ${ }^{5}$ Faculty of Applied Science \& Engineering, University of Toronto, Toronto M5S 1A4, Canada. Instituto de Ciências Biomédicas, Universidade de São Paulo, São Paulo, Brazil.

Received: 3 November 2014 Accepted: 3 February 2015

Published online: 19 February 2015

\section{References}

1. Beilharz EJ, Williams CE, Dragunow M, Sirimanne ES, Gluckman PD. Mechanisms of delayed cell death following hypoxic-ischemic injury in the immature rat: evidence for apoptosis during selective neuronal loss. Brain Res Mol Brain Res. 1995:29:1-14.

2. Dirnagl U, ladecola C, Moskowitz MA. Pathobiology of ischaemic stroke: an integrated view. Trends Neurosci. 1999;22:391-7.

3. Legos JJ, Tuma RF, Barone FC. Pharmacological interventions for stroke: failures and future. Expert Opin Invest Drugs. 2002;11:603-14.

4. Besancon E, Guo S, Lok J, Tymianski M, Lo EH. Beyond NMDA and AMPA glutamate receptors: emerging mechanisms for ionic imbalance and cell death in stroke. Trends Pharmacol Sci. 2008;29:268-75.

5. Yang XR, Lin MJ, McIntosh LS, Sham JS. Functional expression of transient receptor potential melastatin- and vanilloid-related channels in pulmonary arterial and aortic smooth muscle. Am J Physiol Lung Cell Mol Physiol. 2006;290:L1267-76.

6. Aarts $\mathrm{M}$, lihara $\mathrm{K}$, Wei WL, Xiong ZG, Arundine M, Cerwinski W, et al. A key role for TRPM7 channels in anoxic neuronal death. Cell. 2003;115:863-77.

7. Jiang H, Tian SL, Zeng Y, Li LL, Shi J. TrkA pathway(s) is involved in regulation of TRPM7 expression in hippocampal neurons subjected to ischemic-reperfusion and oxygen-glucose deprivation. Brain Res Bull. 2008;76:124-30,

8. Zhan HQ, Li PF, Li SY, Yan FL, Yang J, Liu RL, et al. Influence of lactuside B on the expression of AQP4 and TRPM7 mRNAs in the cerebral cortex after cerebral ischemia injury. Eur Rev Med Pharmacol Sci. 2014;18:1151-7.

9. Zhang Y, Zhou L, Zhang X, Bai J, Shi M, Zhao G. Ginsenoside-Rd attenuates TRPM7 and ASIC1a but promotes ASIC2a expression in rats after focal cerebral ischemia. Neurol Sci : Off J Ital Neurol Soc Ital Soc Clin Neurophysiol. 2012;33:1125-31.

10. Zhao L, Wang Y, Sun N, Liu X, Li L, Shi J. Electroacupuncture regulates TRPM7 expression through the trkA/PI3K pathway after cerebral ischemiareperfusion in rats. Life Sci. 2007:81:1211-22.

11. Sun HS, Jackson MF, Martin L, Jansen $K$, Teves $L$, Cui $H$, et al. Suppression of hippocampal TRPM7 protein prevents delayed neuronal death in brain ischemia. Nat Neurosci. 2009;12:1300-7.

12. Parnas M, Peters M, Dadon D, Lev S, Vertkin I, Slutsky I, et al. Carvacrol is a novel inhibitor of Drosophila TRPL and mammalian TRPM7 channels. Cell Calcium. 2009:45:300-9.

13. Yu H, Zhang ZL, Chen J, Pei A, Hua F, Qian X, et al. Carvacrol, a foodadditive, provides neuroprotection on focal cerebral ischemia/reperfusion injury in mice. PLoS One. 2012;7:e33584.

14. Vannucci RC. Experimental biology of cerebral hypoxia-ischemia: relation to perinatal brain damage. Pediatr Res. 1990;27:317-26.

15. Vannucci RC. Hypoxic-ischemic encephalopathy. Am J Perinatol. 2000;17:113-20

16. Pulera MR, Adams LM, Liu H, Santos DG, Nishimura RN, Yang F, et al. Apoptosis in a neonatal rat model of cerebral hypoxia-ischemia. Stroke: J Cereb Circ. 1998;29:2622-30.

17. Bonfoco E, Krainc D, Ankarcrona M, Nicotera P, Lipton SA. Apoptosis and necrosis: two distinct events induced, respectively, by mild and intense 
insults with N-methyl-D-aspartate or nitric oxide/superoxide in cortical cell cultures. Proc Natl Acad Sci U S A. 1995;92:7162-6.

18. Sidhu RS, Tuor UI, Del Bigio MR. Nuclear condensation and fragmentation following cerebral hypoxia-ischemia occurs more frequently in immature than older rats. Neurosci Lett. 1997;223:129-32.

19. Manabat C, Han BH, Wendland M, Derugin N, Fox CK, Choi J, et al. Reperfusion differentially induces caspase-3 activation in ischemic core and penumbra after stroke in immature brain. Stroke: J Cereb Circ. 2003:34:207-13.

20. Gross A, MCDonnell JM, Korsmeyer SJ. BCL-2 family members and the mitochondria in apoptosis. Gene Dev. 1999;13:1899-911.

21. Linnik MD, Zahos P, Geschwind MD, Federoff HJ. Expression of bcl-2 from a defective herpes simplex virus-1 vector limits neuronal death in focal cerebral ischemia. Stroke: J Cereb Circ. 1995;26:1670-4. discussion 1675.

22. Wang $X$, Han W, Du X, Zhu C, Carlsson Y, Mallard C, et al. Neuroprotective effect of Bax-inhibiting peptide on neonatal brain injury. Stroke: J Cereb Circ. 2010;41:2050-5.

23. Zhao H, Sapolsky RM, Steinberg GK. Phosphoinositide-3-kinase/akt survival signal pathways are implicated in neuronal survival after stroke. Mol Neurobiol. 2006;34:249-70.

24. Giffard RG, Monyer H, Christine CW, Choi DW. Acidosis reduces NMDA receptor activation, glutamate neurotoxicity, and oxygen-glucose deprivation neuronal injury in cortical cultures. Brain Res. 1990;506:339-42.

25. Rice 3rd JE, Vannucci RC, Brierley JB. The influence of immaturity on hypoxic-ischemic brain damage in the rat. Ann Neurol. 1981;9:131-41.

26. Cho KO, Lee KE, Youn DY, Jeong KH, Kim JY, Yoon HH, et al. Decreased vulnerability of hippocampal neurons after neonatal hypoxia-ischemia in bis-deficient mice. Glia. 2012;60:1915-29.

27. Fan X, Heijnen CJ, van der Kooij M, Groenendaal F, van Bel F. Beneficial effect of erythropoietin on sensorimotor function and white matter after hypoxia-ischemia in neonatal mice. Pediatr Res. 2011;69:56-61.

28. Alibrahim A, Zhao LY, Bae CY, Barszczyk A, Sun CL, Wang GL, et al. Neuroprotective effects of volume-regulated anion channel blocker DCPIB on neonatal hypoxic-ischemic injury. Acta Pharmacol Sin. 2013;34:113-8.

29. Sun H-S, Xu B, Chen W, Xiao A, Turlova E, Alibraham A, et al. Neuronal KATP channels mediate hypoxic preconditioning and reduce subsequent neonatal hypoxic-ischemic brain injury. Exp Neurol 2014: In Press, Accepted Manuscript.

30. Friedman M. Chemistry and multibeneficial bioactivities of carvacrol (4-isopropyl-2-methylphenol), a component of essential oils produced by aromatic plants and spices. J Agric Food Chem. 2014;62:7652-70.

31. Braun LD, Cornford EM, Oldendorf WH. Newborn rabbit blood-brain barrier is selectively permeable and differs substantially from the adult. J Neurochem. 1980;34:147-52.

32. Olesen SP. Rapid increase in blood-brain barrier permeability during severe hypoxia and metabolic inhibition. Brain Res. 1986;368:24-9.

33. Romijn HJ, Voskuyl RA, Coenen AM. Hypoxic-ischemic encephalopathy sustained in early postnatal life may result in permanent epileptic activity and an altered cortical convulsive threshold in rat. Epilepsy Res. 1994;17:31-42.

34. Deng W, Lu H, Teng J. Carvacrol attenuates diabetes-associated cognitive deficits in rats. J Mol Neurosci: MN. 2013;51:813-9.

35. Song X, Chen A, Liu Y, Wang XB, Zhou Y, Liu L, et al. Carvacrol pretreatment attenuates myocardial oxidative stress and apoptosis following myocardial ischemia-reperfusion in mice. Nan Fang Yi Ke Da Xue Xue Bao = J Southern Med Univ. 2013;33:1624-7.

36. Zhu C, Hallin U, Ozaki Y, Grander R, Gatzinsky K, Bahr BA, et al. Nuclear translocation and calpain-dependent reduction of $\mathrm{BCl}-2$ after neonatal cerebral hypoxia-ischemia. Brain Behav Immun. 2010;24:822-30.

37. Chien CT, Chang TC, Tsai CY, Shyue SK, Lai MK. Adenovirus-mediated bcl-2 gene transfer inhibits renal ischemia/reperfusion induced tubular oxidative stress and apoptosis. Am J Transplant: Off J Am Soc Transplant Am Soc Transplant Surg. 2005;5:1194-203.

38. Gibson ME, Han BH, Choi J, Knudson CM, Korsmeyer SJ, Parsadanian M, et al. BAX contributes to apoptotic-like death following neonatal hypoxia-ischemia: evidence for distinct apoptosis pathways. Mol Med. 2001;7:644-55.

39. Su LT, Agapito MA, Li M, Simonson WT, Huttenlocher A, Habas R, et al. TRPM7 regulates cell adhesion by controlling the calcium-dependent protease calpain. J Biol Chem. 2006;281:11260-70.

40. Gill R, Soriano M, Blomgren K, Hagberg H, Wybrecht R, Miss MT, et al. Role of caspase-3 activation in cerebral ischemia-induced neurodegeneration in adult and neonatal brain. J Cereb Blood Flow Metab: Off J Int Soc Cereb Blood Flow Metab. 2002;22:420-30.
41. Zhu HC, Gao XQ, Xing Y, Sun SG, Li HG, Wang YF. Inhibition of caspase-3 activation and apoptosis is involved in 3-nitropropionic acid-induced ischemic tolerance to transient focal cerebral ischemia in rats. J Mol Neurosci: MN. 2004;24:299-305.

42. Haylor UL, Harris KP, Nicholson ML, Waller HL, Huang Q, Yang B. Atorvastatin improving renal ischemia reperfusion injury via direct inhibition of active caspase-3 in rats. Exp Biol Med (Maywood). 2011;236:755-63.

43. Xiong T, Tang J, Zhao J, Chen H, Zhao F, Li J, et al. Involvement of the Akt/ GSK-3beta/CRMP-2 pathway in axonal injury after hypoxic-ischemic brain damage in neonatal rat. Neuroscience. 2012;216:123-32.

44. Zotti M, Colaianna M, Morgese MG, Tucci P, Schiavone S, Avato P, et al. Carvacrol: from ancient flavoring to neuromodulatory agent. Molecules. 2013;18:6161-72.

45. Monzote L, Stamberg W, Staniek K, Gille L. Toxic effects of carvacrol, caryophyllene oxide, and ascaridole from essential oil of Chenopodium ambrosioides on mitochondria. Toxicol Appl Pharmacol. 2009;240:337-47.

46. Wei WL, Sun HS, Olah ME, Sun X, Czerwinska E, Czerwinski W, et al. TRPM7 channels in hippocampal neurons detect levels of extracellular divalent cations. Proc Natl Acad Sci U S A. 2007:104:16323-8.

47. Qi X, Hosoi T, Okuma Y, Kaneko M, Nomura Y. Sodium 4-phenylbutyrate protects against cerebral ischemic injury. Mol Pharmacol. 2004;66:899-908.

48. Lubics A, Reglodi D, Tamas A, Kiss P, Szalai M, Szalontay L, et al. Neurological reflexes and early motor behavior in rats subjected to neonatal hypoxic-ischemic injury. Behav Brain Res. 2005;157:157-65.

49. Shen Y, Isaacson RL, Smotherman WP. The behavioral and anatomical effects of prenatal umbilical cord clamping in the rat and their alteration by the prior maternal administration of nimodipine. Restor Neurol Neurosci. 1991;3:11-22.

50. Rha DW, Kang SW, Park YG, Cho SR, Lee WT, Lee JE, et al. Effects of constraint-induced movement therapy on neurogenesis and functional recovery after early hypoxic-ischemic injury in mice. Dev Med Child Neurol. 2011;53:327-33.

51. Chen WL, Huang XQ, Zhao LY, Li J, Chen JW, Xiao Y, et al. Involvement of Kv1.5 protein in oxidative vascular endothelial cell injury. PLoS One. 2012; $7:$ :e49758.

52. Chen WL, Qian Y, Meng WF, Pang JY, Lin YC, Guan YY, et al. A novel marine compound xyloketal B protects against oxidized LDL-induced cell injury in vitro. Biochem Pharmacol. 2009;78:941-50.

\section{Submit your next manuscript to BioMed Central and take full advantage of:}

- Convenient online submission

- Thorough peer review

- No space constraints or color figure charges

- Immediate publication on acceptance

- Inclusion in PubMed, CAS, Scopus and Google Scholar

- Research which is freely available for redistribution

Submit your manuscript at www.biomedcentral.com/submit
C BioMed Central 